\title{
Risk algorithms that include pathology adjustment for HER2 amplification need to make further downward adjustments in likelihood scores
}

\author{
D. G. Evans $s^{1,2,3,4}$ - E. R. Woodward ${ }^{1,3}$ - S. J. Howell ${ }^{2,4,5}$ - S. Verhoef ${ }^{3}$. \\ A. Howell ${ }^{2,4}$ - F. Lalloo $^{3}$
}

Published online: 31 October 2016

(c) The Author(s) 2016. This article is published with open access at Springerlink.com

\begin{abstract}
To assess the need for adjustment in the likelihood of germline BRCA1/2 mutations in women with HER $2+$ breast cancers. We analysed primary mutation screens on women with breast cancer with unequivocal HER2 overexpression and assessed the likelihood of $B R C A 1 / B R C A 2$ mutations by age, oestrogen receptor status and Manchester score. Of 1111 primary BRCA screens with confirmed HER2 status only 4/161 (2.5\%) of women with HER2 amplification had a BRCA1 mutation identified and 5/161 (3.1\%) a BRCA2 mutation. The pathology adjusted Manchester score between 10 and $19 \%$ and $20 \%+$ thresholds resulted in a detection rate of only 6.5 and $15 \%$ respectively. BOADICEA examples appeared to make even less downward adjustment. There is a very low detection rate of $B R C A 1$ and $B R C A 2$ mutations in women with HER2 amplified breast cancers. The Manchester score
\end{abstract}

D. G. Evans

gareth.evans@cmft.nhs.uk

1 Manchester Centre for Genomic Medicine, St Mary's Hospital, Manchester Academic Health Sciences Centre (MAHSC), Institute of Human Development, University of Manchester, Manchester M13 9WL, UK

2 Genesis Breast Cancer Prevention Centre, University Hospital of South Manchester NHS Trust, Wythenshawe, Manchester M23 9LT, UK

3 Manchester Centre for Genomic Medicine, St Mary's Hospital, Manchester Academic Health Sciences Centre (MAHSC), Central Manchester University Hospitals NHS Foundation Trust, Manchester M13 9WL, UK

4 Manchester Breast Centre, School of Molecular and Clinical Cancer Sciences, The University of Manchester, Manchester M20 4BX, UK

5 Department of Medical Oncology, The Christie, Manchester M20 4BX, UK and BOADICEA do not make sufficient downward adjustment for HER2 amplification. For unaffected women, assessment of breast cancer risk and BRCAl/2 probability should take into account the pathology of the most relevant close relative. Unaffected women undergoing mutation testing for BRCA1/2 should be advised that there is limited reassurance from a negative test result if their close relative had a HER2+ breast cancer.

Keywords BRCA1 - BRCA2 - Breast cancer - HER2 . Oestrogen $\cdot$ Manchester score

\section{Introduction}

Although the pathology of breast cancers associated with $B R C A 1$ and to a lesser extent $B R C A 2$ is well documented [1-3], the likelihood of identifying a BRCA1 or BRCA2 mutation in HER2 amplified breast cancer is not well described. Most risk algorithms for assessing the likelihood of a $B R C A 1 / 2$ mutation were developed before the ability to adjust for pathology, particularly relevant for $B R C A 1$ where the majority of breast cancers are high grade and triple negative (estrogen receptor (ER), progesterone receptor (PR) and HER2 negative) [4-8]. The speed and reduced expense of modern $B R C A$ mutation screening may lead to a perceived reduction in the importance of mutation likelihood assessment. However, difficulties in interpretation of mutation testing arise when a variant of uncertain significance (VUS) is found. Furthermore many countries still utilise likelihood thresholds for a sample to be tested, which in the UK remains at 10\% [9]. Until there are licensed medications, approved by healthcare systems, for precision medicine approaches for breast cancer related to $B R C A 1 / 2$ such as PARPi, these thresholds are likely to 
remain. Knowing the a priori likelihood of an individual having a pathogenic mutation is helpful in interpreting VUS reports [3] that affect around 5\% of tested individuals. More importantly, for those individuals testing negative for a BRCA1/2 mutation screen who do not have cancer, and are from a family without testing of an affected member, it is not possible to assess the degree of reassurance of a negative test without knowledge of the likelihood that their affected relative was a $B R C A 1$ or $B R C A 2$ mutation carrier. Attempts have been made to incorporate breast pathology into risk algorithms such as the Manchester scoring system [10] and BOADICEA [11, 12] and these improve the accuracy of likelihood estimates and risk thresholds $[10,13]$. Periodic revisions of these scoring systems are useful, as most 'classical' high penetrance BRCA mutation carrier families will have been identified, leaving less classical phenotypes to be uncovered. Therefore incorporating additional data, such as receptor status, is worthwhile to develop and update the chance algorithms. Partly because of its relatively recent introduction, data on HER2 remains relatively scarce. We have therefore interrogated our data on $B R C A 1 / 2$ primary mutation screens in women with invasive breast cancer and our BRCA1 and BRCA2 family register service databases to address the question of breast pathology in primary screens and of the proportion of BRCA1 and BRCA2 breast cancers that are triple negative or HER2 amplified [14]. We also subdivided HER2 amplified cancers by ER expression.

\section{Methods}

Women with breast cancer have been tested for BRCAl/2 mutations in Manchester since 1996. Data on women with breast cancer who had undergone BRCAl/2 testing were obtained from those undergoing primary full screens for BRCA1/2 mutations with sequencing of all exons and Multiple Ligation dependent Probe Amplification (MLPA) to assess for whole or multiple exon deletions or duplications as previously described [10]. Only women with confirmed pathogenic mutations were included as $B R C A I$ or $B R C A 2$ positive. Whilst risk thresholds for testing were quite stringent with a 20\% threshold for testing until 2013, the majority of women with at least a $10 \%$ probability for a BRCA1/2 mutation and several at less than this had access to testing through research protocols.

All primary breast cancers occurring after 1990 (including contralateral) when hormone receptor testing started to be carried out routinely were included. HER2 testing did not become routine in the UK until around 2005. Data were collected prospectively on all women with breast cancer tested for BRCA1/2 from 2005 and retrospectively for cases tested before that date. The study represents a semi-prospective consecutive series of women with breast cancer where HER2 status was assessable.

\section{Confirmation of HER2 positivity}

HER2 status was assessed from local pathology reports and defined as positive if (1)- scored $3+$ by immunohistochemistry or (2) amplified by Fluorescent (or other) In Situ Hybridisation (ISH) with a HER2:CEP17 ratio of $>2.0$ or HER2 amplicon $>6$ if no CEP17 probe was employed. All negative, unconfirmed and borderline cases were excluded including those where tumours scored IHC $2+$ from the pathology report and no supplementary FISH report was available.

\section{Confirmation of ER negativity}

Breast cancers were classified as ER-ve based on pathology reports from clinical records and cancer registry data. ER was assessed as either a percentage staining $(0-100 \%)$ or as a quickscore (QS) (0-8). A tumor was considered ER positive with a QS of 6-8 and or staining of $>10 \%$. ER negativity was confirmed if QS was $<4$ and or ER was $<10 \%$. Tumors with intermediate scores were excluded.

\section{Confirmation of triple negativity}

From 2005, PR was also routinely tested and classification was identical to that of ER. PR positivity was not recorded on the mutation database but if ER and HER2 were negative and PR had a QS of $<4$ and or percentage was $<10 \%$, tumors were considered triple negative for the database.

Manchester scores were derived by summating scores for BRCA1 and BRCA2 for each breast, ovarian or prostate cancer primary in the same lineage. An adjustment of -4 points was made for a HER $2+$ breast cancer and +4 for a grade 3 triple negative breast cancer as previously described [10]. Two sided Chi-square tests with Fisher's exact correction were used to compare proportions.

Ethics approval for the study was through the North Manchester Research (08/H1006/77) and University of Manchester ethics committees (08229).

\section{Results}

There were 1134 women with breast cancer with verified HER2 and ER status who had undergone full mutation screening of BRCAI and BRCA2 (Table 1). Included were 619 of the total of $803(77 \%)$ samples that had BRCA tested in Manchester in the last 4 years (since 01/11/2011). Twenty-three women with breast cancer were excluded from the main analysis as their tumours were ER negative 
Table 1 HER2 and ER status in primary BRCA screens

\begin{tabular}{|c|c|c|c|c|c|c|}
\hline & BRCA1 & $B R C A 2$ & negative & Total & $\begin{array}{l}\text { Proportion with } \\
B R C A \text { mutations }(\%)\end{array}$ & $\begin{array}{l}95 \% \text { Confidence } \\
\text { intervals }(\%)\end{array}$ \\
\hline $\mathrm{HER} 2+\mathrm{MS}<14$ & 0 & 1 & 81 & 82 & 1.2 & $0.0-3.6$ \\
\hline HER2+ MS 14-20 & 1 & 2 & 43 & 46 & 6.5 & $0.0-13.7$ \\
\hline ER + HER2 + 14-20 & 1 & 1 & 37 & 39 & 5.1 & $0.0-12.1$ \\
\hline HER2 + MS 20+ & 3 & 2 & 28 & 33 & 15 & $2.9-27.4$ \\
\hline ER + HER2 + MS 20+ & 0 & 1 & 22 & 23 & 4.4 & $0-12.7$ \\
\hline Total HER2+ & 4 & 5 & 152 & 161 & 5.6 & $2.0-9.2$ \\
\hline ER - HER $2-<50$ years & 103 & 25 & 213 & 341 & 37.5 & $32.4-42.7$ \\
\hline ER - HER2 $-50+$ & 14 & 9 & 61 & 84 & 27.3 & $17.8-36.9$ \\
\hline ER - HER $2-$ sporadic $<50$ years & 6 & 2 & 86 & 94 & 8.5 & $2.9-14.2$ \\
\hline ER - HER2 - MS $<14$ & 6 & 0 & 79 & 85 & 7.0 & $1.6-12.5$ \\
\hline ER- HER2- MS 14-20 & 15 & 10 & 100 & 125 & 20.0 & $13.0-27.0$ \\
\hline ER - HER2- MS 20+ & 96 & 24 & 95 & 215 & 55.8 & $49.2-62.4$ \\
\hline ER - HER2 - MS 30+ & 51 & 8 & 12 & 71 & 83.1 & $74.4-91.8$ \\
\hline Total ER - HER2- ${ }^{\mathrm{a}}$ & 117 & 34 & 274 & 425 & 35.5 & $30.9-40.0$ \\
\hline
\end{tabular}

${ }^{a}$ This excludes 23 women with ER- PR+ HER2- breast cancers. None had BRCA1/2 mutations

$M S$ manchester score

and PR positive, resulting in 1111 eligible women with ER/ PR negative tumour with a known HER2 status. The majority $(n=101)$ of the 184 women in the most recent era (2010-2015) without HER2 status were diagnosed with breast cancer before 2005, $78(9.8 \%)$ had no available pathology report and the remaining five $(0.6 \%)$ were HER2 $2+$ without available FISH testing from clinical notes. Of the 1111 women, 161 (14.5\%) had HER2 amplified breast cancer. 114/161 (71\%) were ER positive. Only 9/161 $(5.6 \%)$ of BRCA tests in HER2+ women identified a pathogenic mutation. Five of $114(4.4 \%)$ of ER+ HER2+ cancers had mutations (4 BRCA2) and 4/45 (9\%) of ERHER2 + (3 BRCA1). In contrast, of 425 screens in women with triple negative breast cancer, $151(35.5 \%)$ resulted in positive BRCA tests with $117(27.5 \%)$ having BRCA1 mutations and $34(8.0 \%)$ BRCA2 mutations. Even with a combined pathology adjusted Manchester score [10] of 20 or higher indicating at least a $20 \%$ likelihood of a BRCA1/2 mutation only $5 / 33(15 \%)$ women with HER2+ breast cancer had a mutation identified. However, this was $4 / 10$ $(40 \%)$ of ER - HER + cancers and only 1/23 (4.4\%; $95 \%$ CI $0-12.7 \%)$ of ER+ HER2 - cancers $(p=0.02)$. It was not really possible to assess the $10 \%$ threshold with a Manchester score of 15-19 as only seven breast cancers that were ER - HER2 + were tested one of which $(14 \%)$ had a BRCA2 mutation. None of 22 sporadic HER2+ breast cancers had a BRCA1/2 mutation, but $2 / 13(15 \%)$ diagnosed $<35$ years had a TP53 mutation. In contrast $120 / 215(55.8 \%)$ of those with triple negative breast cancer and a Manchester score above 20 had a mutation identified, rising to $83 \%$ in women with a Manchester score of 30 or higher.

Interestingly, the $10 \%$ threshold was not met with triple negative breast cancers with a Manchester score of $<14(6 /$ $85=7.0 \%$ ), nor with sporadic triple negative cases of breast cancer aged $<50$ years at diagnosis $(8 / 94=8.5 \%)$.

\section{Discussion}

This present study has demonstrated a very low frequency of detection of BRCA1 and BRCA2 mutation carriers amongst primary screens of women with HER2 amplified breast cancers, particularly those with ER+ ve tumours. Overall, these low rates do not appear to be fully accounted for in the pathology adjusted Manchester scoring system with the $10 \%$ risk combined threshold not being met in women with scores of 14-19 and the 20\% threshold not being met in women with Manchester scores of 20 or higher (5/33-15\%), excepting those that were ER-ve. In practice, this suggests that women with ER+ HER2 positive breast cancers should be advised that they are unlikely to harbour a $B R C A 1 / 2$ mutation unless there are other very suggestive features in their own personal or family histories (other more typical breast cancer or ovarian cancer). Additionally, women who are offered testing whose mother or sister had a HER2 positive breast cancer will get little reassurance regarding breast cancer risk from a negative test unless there is also a strong additional family history suggestive of BRCA1/2. This is because these women will 
still have an increased risk of breast cancer, due to the likely presence of other breast cancer genes within their families.

Whilst the low level of detection of BRCA1 and BRCA2 mutations amongst individuals with HER2+ breast cancers is clearly important in assessing carrier likelihood, the presence of a triple negative breast cancer clearly increases the likelihood of identifying a $B R C A 1 / 2$ mutation. It has been suggested that all women with triple negative breast cancer aged $<50$ years should be tested for BRCA1/2 mutations based on the overall detection rate being above $10 \%[15,16]$. However, this does not take into account the fact that the $10 \%$ threshold is not clearly reached in women with an isolated breast cancer and no family history [15], In a large study of 1824 cases [15], only 18/209 (8.6\%) women with sporadic triple negative breast cancer diagnosed aged 40-49 years had an identifiable mutation in $B R C A 1 / 2$. This is supported by a detection rate of only $8 / 94(8.5 \%)$ in sporadic triple negative cases $<50$ years in the present study although the 95\% confidence intervals do overlap with $10-8.5 \%$ (95\% CI 2.9-14.2\%). Nonetheless, sporadic cases under 40 years do meet the $10 \%$ threshold [17] with 23/149 (18\%) of 35-39 year old sporadic cases having a mutation and 23\% $(18 / 91)$ of those $<35$ years of age at diagnosis. Clearly $8.6 \%$ is still close to the $10 \%$ threshold (the upper $95 \% \mathrm{CI}$ is $12.4 \%$ ) and many centres may consider it simpler just to test all cases $<50$ years. Additionally, testing women with few unaffected female relatives and in particular, adopted women seems appropriate as the $10 \%$ threshold may be reached in these groups.

At the other end of the spectrum, unaffected women whose mother or sister with high grade triple negative breast cancer is unavailable for testing and whose family history is strongly predictive of a BRCAl/2 mutation, (such as a BOADICEA likelihood in the relative of $>80 \%$ or a pathology adjusted Manchester score of 30 or higher) should be reassured by a negative test. The majority of their inherited risk would be due to a discoverable BRCAl/ 2 mutation and the negative test will greatly reduce their risk of breast and ovarian cancer, due to the high sensitivity of current BRCA testing [18].

An example pedigree (Fig. 1) is given to show the effects of using or not using HER2 status in assessing breast cancer risk for a 25-year old unaffected woman, with an affected mother and maternal aunt with breast cancers at age 35 years. Using the Manchester score [10], a grade 3 triple negative breast cancer would add 4 points to the Manchester score of 16 to reach 20 points. A HER2+ breast cancer in the mother would reduce the score to 12 points. An unadjusted Manchester score would be equivalent to a $10 \%$ probability of $B R C A 1 / 2$ in the mother. This would rise to $20 \%$ with Triple negative and reduce to $5 \%$ if mother had a HER2 + tumour. The likelihoods are halved in the proband. The attributable risks of breast cancer using $80 \%$ penetrance would be only $2 \%$ if mother was HER $2+$ rising to $8 \%$ if mother was triple negative (Table 2). Readouts for Tyrer-Cuzick (changes to BRCA1/2 probability inferred) and BOADICEA [12] and BRCAPRO [5] (both with inbuilt pathology adjustments) are shown in Table 2. Apart from with Tyrer-Cuzick, which does not have an inbuilt adjustment for pathology, the reduction in breast cancer risk is only about $2 \%$ for testing negative in the proband when the mother was HER2+. This changes to a $5.7-10.5 \%$ reduction if the mother had triple negative breast cancer. With BOADICEA the BRCA1/2 probability in the proband falls from 6.5 to $3.6 \%$ with HER2+ breast cancer in mother and rises to $12.3 \%$ with triple negative. A negative $B R C A 1 / 2$ mutation test only drops absolute breast cancer risk by $1.7 \%$ if mother was Her $2+$ but by nearly $6 \%$ when triple negative. These may underestimate the reductions due to the default BRCA sensitivities being only 70 and $80 \%$ for $B R C A 1 / 2$ respectively, which is below the sensitivity of at least $84 \%$ (Table 1) for triple negatives with Manchester scores above 30 in this report and our previous identification of BRCA1/2 mutations in 81/94 $(86 \%)$ of breast/ovarian families with Manchester scores of $40+$ [19]. Whilst the downward adjustment for ERHER2+ breast cancers in BOADICEA appears appropriate, the programme currently does not adjust for HER2+ breast cancers when they are also ER+. The downward adjustment for an ER+ HER2+ breast cancer is only from 6.5 to $4.7 \%$ whereas the current report only identified mutations in $4.5 \%$ of ER+ HER2 + breast cancers when the average detection rate in ER+ HER2 - breast cancer was $14.8 \%$ (Table 1). The current data would therefore suggest that further downward adjustment for HER2 amplification is still necessary for ER+ tumours with BOADICEA. Using BRCAPRO (from Cagene v.6) there is a greater adjustment with a $10 \%$ likelihood in the proband for BRCA1/2 dropping to $3.4 \%$ with HER2+ in mother and rising to $22.5 \%$ with triple negative. However, the breast cancer risk readouts for BRCAPRO only include familial risk from $B R C A 1 / 2$ and therefore the reassurance of reducing risks to population levels after negative $B R C A 1 / 2$ testing is inappropriate, [20] as is demonstrated by the far lower breast cancer risk predictions with BRCAPRO compared to Tyrer-Cuzick and BOADICEA. BRCAPRO significantly underestimates breast cancer risk in the familial breast cancer risk setting [20]. Nonetheless BRCAPRO does have a specific readout for ER+ HER2+ breast cancer that is different to ER- HER2+ of $4.8 \%$. Overall an approximate halving of BRCA probability with a HER2 + breast cancer and doubling with a triple negative breast cancer appears to fit the current data.

The present study does have some limitations. The numbers are relatively small compared to large consortia 
Fig. 1 Tyrer-Cuzick risk readout of unadjusted breast cancer risk estimation of case example
Woman's age is 25 years.

Age at menarche is unknown.

No information about childbirth.

Menopause status is unknown.

Height is unknown.

Weight is unknown.

Woman has never used HRT.

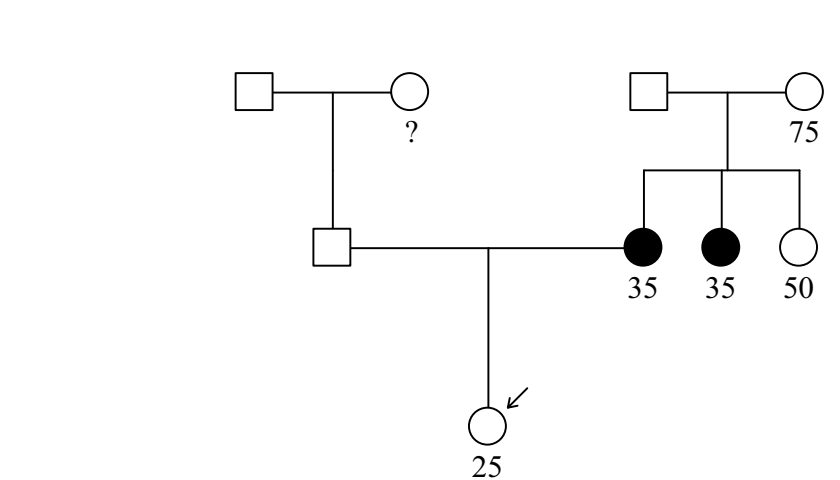

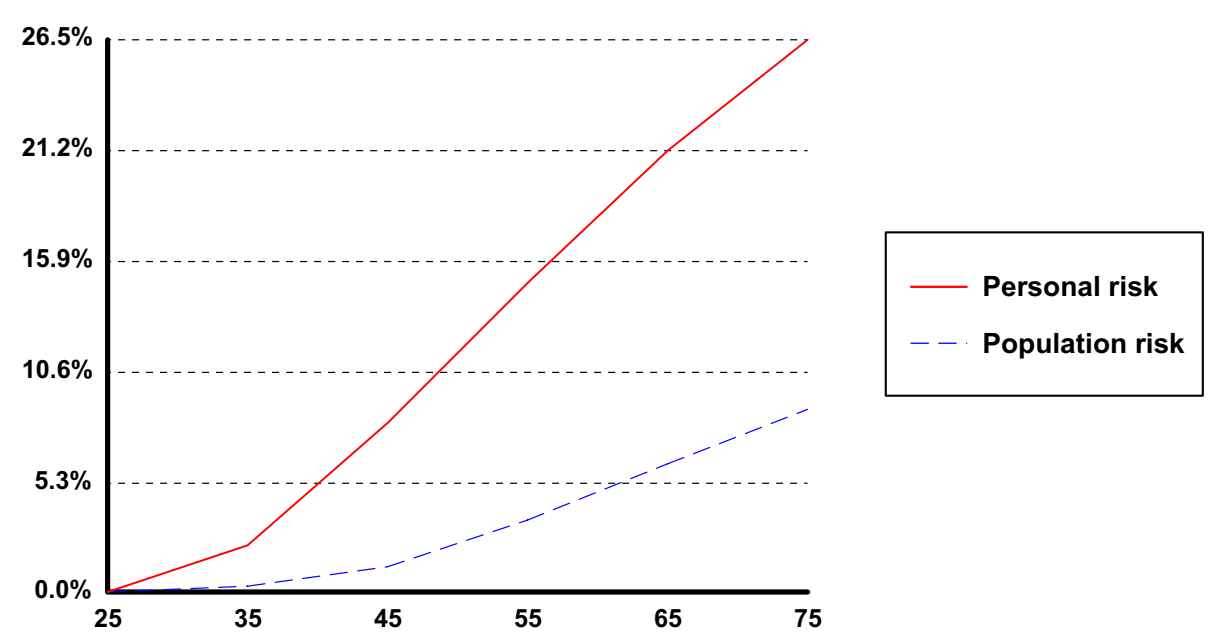

Risk after 10 years is $2.225 \%$.

10 year population risk is $0.226 \%$.

Lifetime risk is $29.16 \%$.

Lifetime population risk is $10.26 \%$.

Probability of a BRCA1gene is $7.126 \%$.

Probability of a BRCA2gene is $6.168 \%$.
[14], but this allows consistency of the approach to classifying HER2 status. In recent times testing of HER2+ samples will have been relatively reduced because of implementing a pathology adjusted Manchester score [10]. This will have boosted numbers with triple negative cancers at the expense of HER2+ cancers thus reducing the overall rate with HER2+ cancers to $13.5 \%$ which is below the overall rate in all breast cancers. It is not clear whether HER2 + breast cancers are more or less likely to have a familial component outside of BRCA1 and BRCA2, although TP53 related breast cancers, which make up a very small proportion of familial breast cancer, are usually HER2 + [21, 22]. Indeed one aspect of the present study is that sporadic HER2+ breast cancer is extremely unlikely to have a $B R C A 1 / 2$ mutation but $<35$ years may well have a TP53 mutation. With more women with breast cancers at early ages undergoing mutation testing to determine treatment even without a family history, extra weight should be given to discussing TP53 in very young sporadic HER2 + breast cancers than the very small possibility of $B R C A 1 / 2$. Although PR was not collected systematically it was when both ER and HER2 were negative. In a large study of 631 breast cancers that were HER2- and ERonly $43(6.8 \%)$ were PR positive [23] similar to the $23 / 438$ $(5.2 \%)$ in the current study.

In conclusion the present study demonstrates the great importance of properly assessing breast cancer HER2 status when determining the likelihood of a BRCA1/2 mutation. Where possible this information should be sought, especially when testing unaffected women whose affected relative with breast cancer is unavailable for genetic testing. Use of well validated programmes that take into 
Table 2 Change in breast cancer risks given to a woman with mother and maternal aunt with breast cancer in their thirties when mother had an $\mathrm{ER}-\mathrm{Her} 2+$ or grade 3 triple negative $(\mathrm{G} 3 \mathrm{TN})$ breast cancer based on BRCA1/2 testing of the unaffected proband

\begin{tabular}{|c|c|c|c|c|}
\hline & $\begin{array}{l}\text { Risk to } 80 \text { years of age prior } \\
\text { to BRCA1/2 testing Not } \\
\text { taking into account histology }\end{array}$ & $\begin{array}{l}\text { Adjusted risk taking into account } \\
\text { change in } B R C A 1 / 2 \text { probability } \\
\text { from histology prior to testing }\end{array}$ & $\begin{array}{l}\text { Final risk based } \\
\text { on negative } \\
\text { BRCA1/2 } \\
\text { testing }\end{array}$ & $\begin{array}{l}\text { Reduction in breast } \\
\text { cancer risk based on } \\
\text { negative testing }(\%)\end{array}$ \\
\hline \multirow[t]{2}{*}{ Tyrer-Cuzick v.6 Her2+ } & BRCA1/2 13\% & $B R C A 1 / 26.5 \%{ }^{\mathrm{b}}$ & $B R C A 1 / 20.5 \%{ }^{\mathrm{a}}$ & \multirow[t]{2}{*}{5} \\
\hline & $29 \%$ BC risk & $24 \%$ BC risk & $19 \% \mathrm{BC}$ risk & \\
\hline \multirow[t]{2}{*}{ Tyrer-Cuzick v.6 G3TN } & BRCA1/2 13\% & $B R C A 1 / 226 \%^{\mathrm{b}}$ & $B R C A 1 / 22 \%^{\mathrm{a}}$ & \multirow[t]{2}{*}{20} \\
\hline & $29 \%$ BC risk & $39 \% \mathrm{BC}$ risk & $19 \% \mathrm{BC}$ risk & \\
\hline \multirow[t]{2}{*}{ BOADICEA HER $2+{ }^{\mathrm{c}}$} & $B R C A 1 / 26.5 \%$ & BRCA1/2 3.6\% & $B R C A 1 / 21 \%^{\mathrm{c}}$ & \multirow[t]{2}{*}{1.7} \\
\hline & $26 \% \mathrm{BC}$ risk & $24 \% \mathrm{BC}$ risk & $22.3 \% \mathrm{BC}$ risk & \\
\hline \multirow[t]{2}{*}{ BOADICEA G3TN } & $B R C A 1 / 26.5 \%$ & $B R C A 1 / 212.3 \%$ & $B R C A 1 / 23.83 \%$ & \multirow[t]{2}{*}{5.8} \\
\hline & $26 \% \mathrm{BC}$ risk & $29.5 \%$ BC risk & $23.7 \% \mathrm{BC}$ risk $^{\mathrm{c}}$ & \\
\hline \multirow[t]{2}{*}{ BRCAPRO Her2+ } & BRCA1/2 10\% & BRCA1/2 3.4\% & $B R C A 1 / 20.2 \%$ & \multirow[t]{2}{*}{1.5} \\
\hline & $17 \% \mathrm{BC}$ risk & $13.7 \% \mathrm{BC}$ risk & $12.2 \% \mathrm{BC}$ risk & \\
\hline \multirow[t]{2}{*}{ BRCAPRO G3TN } & $B R C A 1 / 2 \quad 10 \%$ & $B R C A 1 / 222.5 \%$ & $B R C A 1 / 21.2 \%$ & \multirow[t]{2}{*}{10.5} \\
\hline & $17 \% \mathrm{BC}$ risk & $23.3 \% \mathrm{BC}$ risk & $12.8 \% \mathrm{BC}$ risk & \\
\hline \multirow{2}{*}{$\begin{array}{l}\text { Breast cancer risk } \\
\text { attributable from } B R C A 1 / 2 \\
\text { alone using Manchester } \\
\text { score Her2+ }\end{array}$} & $\begin{array}{l}10 \% \text { likelihood in mother } \\
\text { with MS } 16\end{array}$ & $\begin{array}{l}5 \% \text { likelihood in mother with MS } \\
12\end{array}$ & & \multirow[t]{2}{*}{2} \\
\hline & $4 \% \mathrm{BC}$ risk & $2 \% \mathrm{BC}$ risk & & \\
\hline \multirow{2}{*}{$\begin{array}{l}\text { Breast cancer risk } \\
\text { attributable from BRCA1/2 } \\
\text { alone using Manchester } \\
\text { score G3TN }\end{array}$} & $\begin{array}{l}10 \% \text { likelihood in mother } \\
\text { with MS } 16\end{array}$ & $\begin{array}{l}20 \% \text { likelihood in mother with MS } \\
20\end{array}$ & & \multirow[t]{2}{*}{8} \\
\hline & $4 \% \mathrm{BC}$ risk & $8 \% \mathrm{BC}$ risk & & \\
\hline
\end{tabular}

BRCAPRO readout from Cagene v.6 https://www4.utsouthwestern.edu/breasthealth/cagene/

G3TN grade 3 triple negative

a This assumes $>90 \%$ sensitivity of $B R C A 1 / 2$ testing

b This assumes a halving of BRCA1/2 probability with Her2+ and doubling with G3TN and no change of BRCAX status

c Although the current online BOADICEA (https://pluto.srl.cam.ac.uk/) does include HER" and ER status HER" is only taken into account when ER is negative. BRCA1/2 testing sensitivities are set at 70 and $80 \%$ for BRCA1/2

account the possibility of familial risk other than BRCAl/2 should be used although further adjustments may need to be made before these models fully account for the effects of HER2+ status.

Acknowledgements This research received no specific grant from any funding agency in the public, commercial or not-for-profit sectors. We would like to thank the Genesis Breast Cancer Prevention Appeal and MAHSC. Prof Evans is an NIHR senior investigator.

Author contribution DGE conceived of the study, collected the data with FL, conducted data analysis and statistics. DGE, FL, ERW, SV, $\mathrm{SH}$ and $\mathrm{AH}$ wrote the manuscript, commented on drafts and approved the final version. We are happy to provide anonymised data on request.

\section{Compliance with ethical standards}

Conflict of interest DGE has received a consultancy fee from Astrazeneca. There are no other competing interests.

Open Access This article is distributed under the terms of the Creative Commons Attribution 4.0 International License (http:// creativecommons.org/licenses/by/4.0/), which permits unrestricted use, distribution, and reproduction in any medium, provided you give appropriate credit to the original author(s) and the source, provide a link to the Creative Commons license, and indicate if changes were made.

\section{References}

1. Lakhani SR, Van De Vijver MJ, Jacquemier J et al (2002) The pathology of familial breast cancer: predictive value of immunohistochemical markers estrogen receptor, progesterone receptor, HER-2, and p53 in patients with mutations in BRCA1 and BRCA2. J Clin Oncol 20(9):2310-2318

2. Lakhani SR, Reis-Filho JS, Fulford L et al (2005) Prediction of BRCA1 status in patients with breast cancer using estrogen receptor and basal phenotype. Clin Cancer Res 11(14):5175-5180

3. Spurdle AB, Couch FJ, Parsons MT et al (2014) Refined histopathological predictors of BRCA1 and BRCA2 mutation status: a large-scale analysis of breast cancer characteristics from the BCAC, CIMBA, and ENIGMA consortia. Breast Cancer Res 16(6):3419

4. Evans DG, Graham J, O'Connell S, Arnold S, Fitzsimmons D (2013) Familial breast cancer: summary of updated NICE guidance. BMJ 25(346):f3829. doi:10.1136/bmj.f3829 
5. Parmigiani G, Berry DA, Aquilar O (1998) Determining carrier probabilities for breast cancer susceptibility genes BRCA1 and BRCA2. Am J Human Genet 62:145-148

6. Antoniou AC, Cunningham AP, Peto J et al (2008) The BOADICEA model of genetic susceptibility to breast and ovarian cancers: updates and extensions. Br J Cancer 98(12):2015

7. Tyrer J, Duffy SW, Cuzick J (2004) A breast cancer prediction model incorporating familial and personal risk factors. Stat Med 23(7):1111-1130

8. Evans DG, Eccles DM, Rahman N et al (2004) A new scoring system for the chances of identifying a BRCA1/2 mutation outperforms existing models including BRCAPRO. J Med Genet 41(6):474-480

9. Frank TS, Deffenbaugh AM, Reid JE et al (2002) Clinical characteristics of individuals with germline mutations in BRCA1 and BRCA2: analysis of 10,000 individuals. J Clin Oncol 20(6): 1480-1490

10. Evans DG, Lalloo F, Cramer A et al (2009) Addition of pathology and biomarker information significantly improves the performance of the Manchester scoring system for BRCA1 and BRCA2 testing. J Med Genet 46(12):811-817

11. Mavaddat N, Rebbeck TR, Lakhani SR, Easton DF, Antoniou AC (2010) Incorporating tumor pathology information into breast cancer risk prediction algorithms. Breast Cancer Res 12(3):R28

12. Lee AJ, Cunningham AP, Kuchenbaecker KB, Mavaddat N, Easton DF, Antoniou AC, Consortium of Investigators of Modifiers of BRCA1/2; Breast Cancer Association Consortium (2014) BOADICEA breast cancer risk prediction model: updates to cancer incidences, tumor pathology and web interface. $\mathrm{Br} \mathrm{J}$ Cancer 110(2):535-545

13. Fischer C, Kuchenbäcker K, Engel C et al (2013) Evaluating the performance of the breast cancer genetic risk models BOADICEA, IBIS, BRCAPRO and claus for predicting BRCA1/2 mutation carrier probabilities: a study based on 7352 families from the German hereditary breast and ovarian cancer consortium. J Med Genet 50(6):360-367
14. Mavaddat N, Barrowdale D, Andrulis IL et al (2012) Pathology of breast and ovarian cancers among BRCA1 and BRCA2 mutation carriers: results from the consortium of investigators of modifiers of BRCA1/2 (CIMBA). Cancer Epidemiol Biomark Prev 21(1):134-147

15. Couch FJ, Hart SN, Sharma P et al (2015) Inherited mutations in 17 breast cancer susceptibility genes among a large triple-negative breast cancer cohort unselected for family history of breast cancer. J Clin Oncol 33(4):304-311

16. Robertson L, Hanson H, Seal S et al (2012) BRCA1 testing should be offered to individuals with triple-negative breast cancer diagnosed below 50 years. Br J Cancer 106(6):1234-1238

17. Evans DG, Howell A, Ward D, Lalloo F, Jones JL, Eccles DM (2011) Prevalence of BRCA1 and BRCA2 mutations in triple negative breast cancer. J Med Genet 48(8):520-522

18. Ingham SL, Warwick J, Buchan I et al (2013) Ovarian cancer among 8005 women from a breast cancer family history clinic: no increased risk of invasive ovarian cancer in families testing negative for BRCA1 and BRCA2. J Med Genet 50(6):368-372

19. Smith MJ, Gifford FL, Lalloo F, Newman WG, Evans DG (2012) High sensitivity for BRCA1/2 mutations in breast/ovarian kindreds: are there still other breast/ovary genes to be discovered? Breast Cancer Res Treat 134(2):895-897

20. Amir E, Evans DG, Shenton A, Lalloo F, Moran A, Boggis C, Wilson M, Howell A (2003) Evaluation of breast cancer risk assessment packages in the family history evaluation and screening programme. J Med Genet 40(11):807-814

21. Wilson JR, Bateman AC, Hanson H et al (2010) A novel HER2positive breast cancer phenotype arising from germline TP53 mutations. J Med Genet 47(11):771-774

22. Bougeard G, Renaux-Petel M, Flaman JM et al (2015) Revisiting Li-Fraumeni syndrome from TP53 mutation carriers. J Clin Oncol 33(21):2345-2352

23. Copson E, Eccles B, Maishman T et al (2013) Prospective observational study of breast cancer treatment outcomes for UK women aged 18-40 years at diagnosis: the POSH study. J Natl Cancer Inst 105(13):978-988 